\title{
Logo based Image Copyright Protection using Discrete Wavelet Transform and Fuzzy Inference System
}

\author{
Vaishali S. Jabade \\ Assistant Professor \\ Vishwakarma Institute of Technology, Pune, India
}

\author{
Sachin R. Gengaje, PhD. \\ Professor \\ Walchand Institute of Technology, Solapur, India
}

\begin{abstract}
The digital revolution has resulted in explosion of knowledge. It has encouraged and motivated digitization of the intellectual artefact. This creates issues of piracy and copyright. In this paper, the authors propose a novel logo based watermarking scheme for image copyright protection using fuzzy inference system (FIS), discrete wavelet transform (DWT) and human visual system (HVS). Wavelet transform generates a hierarchical image data structure to represent an image at different resolution levels. The proposed scheme uses fuzzy inference system to adaptively insert logo watermark instead of using uniform thresholding. The weight of watermark is determined by human visual system characteristics and fuzzy inference system. The image is reconstructed by computing inverse discrete wavelet transform to get imperceptible image watermarking. The watermark is then recovered at the receiver end by applying DWT to watermarked image.
\end{abstract}

\section{General Terms}

Copyright Protection, Image Watermarking.

\section{Keywords}

Discrete Wavelet Transform (DWT), Fuzzy Inference System (FIS), Human Visual System (HVS).

\section{INTRODUCTION}

Digital multimedia such as images, texts, music, and videos are often transmitted through open channels of the Internet. Without proper protection mechanism, digital data can be easily copied, modified, tampered, or forged during multimedia transmission. Protecting the integrity, validity and ownership of digital multimedia has become an important issue today. Image watermarking is an important aspect of image copyright protection. The other applications of image watermarking include broadcast monitoring, content authentication, copy control, usage control, fingerprinting etc. Copyright protection of digital images is receiving widespread attention in recent years as digital images are very much susceptible to copyright infringements. Digital image watermarking is an effective tool for protecting images from such copyright infringement. It discourages unauthorized copying of images. The information extracted from watermarked image is used to identify rightful ownership. Watermarking an image is a process of altering pixel values of an image in a manner so that there is only a slight difference between original image and watermarked image. The difference may be perceptually visible or invisible $[1,2]$.

The digital watermark is perceptible or imperceptible identification code that is embedded into host image; which uniquely identifies its ownership. In copyright protection application, digital watermark can be ownership identifier, transaction date, serial number, logo, trademark or copyright information. Invisible watermarks change the image in spatial or frequency domains in a way, which only machines can detect. One can extract watermark in order to verify ownership.

Image watermarking can also be used as a way to transport information secretly or to protect integrality of cover image itself. Conventionally, most of the watermarking schemes embedded pseudo-random sequence of independently distributed numbers in the image. Though these watermarks can be extracted, it is very difficult for distinct identity of the same. This leads to limitation on effectiveness and use for copyright protection of images. Thus there is a need to develop a method to embed readable watermark such as text or logo in the images that can be easily identified upon extraction. In this paper, copyright protection scheme in wavelet domain is developed. The novel approach allows image owner to adjust weight of watermark using FIS and HVS, so that imperceptibility along with robustness of watermark can be enhanced.

\subsection{Image Copyright Protection Attributes}

Different applications of image watermarking demand different attributes. Requirements of image watermarking vary depending on the image watermarking applications and result in various design issues. Properties of effective image watermarking for copyright protection are as follows:

\subsubsection{Robustness}

It is the ability of watermark to resist various image processing and geometric attacks. Image processing attacks include JPEG Compression, salt and pepper noise, Gaussian noise, filtering etc. Geometric attacks include rotation, scaling, cropping etc. [3-5] Geometric attacks distort the watermark through spatial or temporal alterations of the watermarked image [6, 7]. For copyright protection application, robustness of watermark is primary requirement. For most of the watermarking methods, robustness against geometric attacks is vital for detection of watermark. Geometric attacks do not cause serious visual distortion. But they can severely affect watermark detection by changing detected position of the embedded watermark.

\subsubsection{Imperceptibility}

It represents transparency of watermark such that it is perceptually invisible. Modification of the host image must be inconspicuous after watermarks are embedded. Invisible watermark is imperceptible copyright information. It is hidden directly in media content in such a manner that cannot be removed by the user. But this information can be extracted or read by the appropriate party [8-10].

\subsubsection{Blindness}

The ability to recover watermark in the absence or presence of host image indicates blindness. Non blind or private image watermarking requires original image required to extract the watermark enhancing security aspect. On the other hand, blind 
or public image watermarking does not require original image for detection reducing storage requirements.

\subsubsection{Un-ambiguity}

Un-ambiguity refers to clear verification of extracted watermark [11]. The extracted logo must be clear enough so that it can indicate ownership of the host image exactly.

\subsubsection{Computational Complexity}

The amount of time watermarking algorithm takes to encode and decode is another consideration. More computations may be needed to ensure digital watermark security and validity once it is received. [12].

\section{PROPOSED APPROACH}

The image watermarking method comprises of watermark embedding and watermark extraction process. The proposed scheme uses imperceptible non blind watermarking comprising of aspects of Discrete Wavelet Transform (DWT), Human Visual System (HVS) and Fuzzy Inference System (FIS) as discussed.

\subsection{Discrete Wavelet Transform (DWT)}

Wavelet transform is a mathematical tool for hierarchically decomposing an image. Wavelets allow image to be described in terms of a coarse overall shape and details that range from broad to narrow because of multi-resolution approach. Hence wavelets reflect anisotropic properties of HVS more precisely. It is a special case of sub band transform with filter bank. The filter bank includes decomposition filter, reconstruction filter, down-sampler and up-sampler [13, 14]. Further, JPEG200, new standard for still image compression is based on DWT. Image watermarking achieves better robustness if watermark domain and compression domain are same. DWT can enhance robustness of the watermark against intentional and unintentional attacks. Thus, DWT based watermarking methods are extensively used. [15-20].

\subsection{Human Visual System (HVS)}

Psycho-visual studies have shown that HVS has a general band pass characteristics. The sensitivity of human vision is different in various spatial frequencies (frequency bands). HVS research offers mathematical models of human vision. A number of factors affect noise sensitivity of human vision like luminance, frequency band, texture, proximity to an edge etc. $[21,22]$

The wavelet based algorithm uses human visual characteristics to exploit human eye limitation to embed strong watermark which maintains perceptual invisibility. The principal advantage of using HVS is adaptive image watermarking. HVS exhibits following characteristics:

1. Human vision is less sensitive to noise in high resolution bands and bands having orientation of $\pm 45^{\circ}$.

2. Human vision is less sensitive to noise in areas where image brightness is high or low.

3. Human vision is less sensitive to noise in highly textured areas but more sensitive near edges [23-25].

\subsection{Fuzzy Inference System (FIS)}

Fuzzy logic is a multi-valued logic and is almost synonymous with fuzzy set theory which relates to classes of objects with un-sharp boundaries in which membership is a matter of degree. Fuzzy set theory has potential capability to represent input and output relationships of dynamic systems. A fuzzy inference system is necessary to provide reasoning with vague and uncertain information. The basic concept underlying fuzzy logic is that variable values are words or linguistic variables, rather than numbers. Although words are inherently less precise than numbers, their use is closer to human intuition. Computing with words exploits tolerance for imprecision and thereby lowers cost of solution. Thus fuzzy logic has predicates with fractional values rather than simply being true or false values. Fuzzy inference system used is shown in figure 1. Fuzzifier accepts crisp input and converts it into fuzzy output. Fuzzy inference uses image features like brightness, contrast and texture along with fuzzy rules to aggregate into fuzzy output. Defuzzifier converts fuzzy output back to crisp output [26-33].

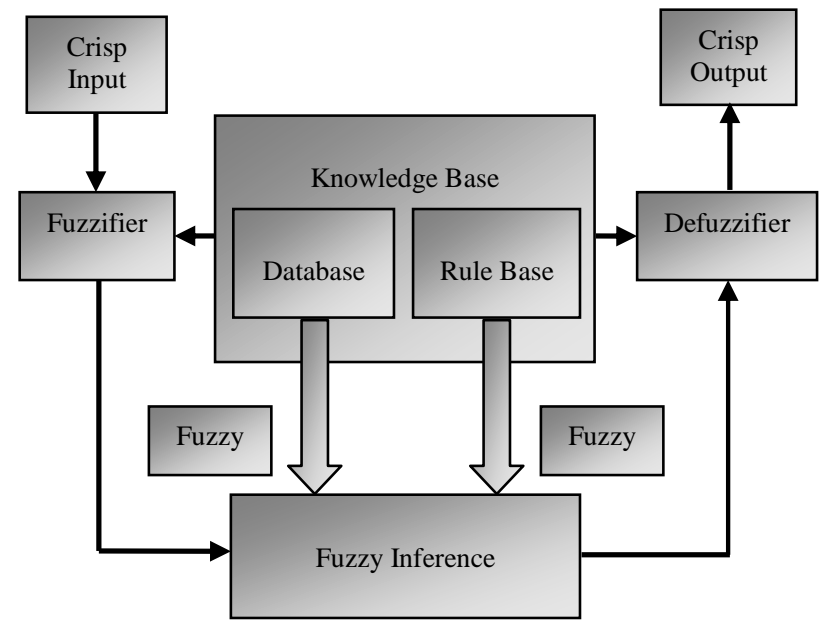

Fig.1. Block Diagram for Watermark Embedding

\section{WATERMARK EMBEDDING PROCESS}

The watermark embedding process first decomposes original image into three levels using DWT. The local characteristics of the image are subsequently extracted using the model of HVS. The quantization steps for DWT coefficients are then computed using FIS in order to generate watermark weighting function. Watermark is then inserted in sub-bands. The functional block diagram of proposed watermark embedding process is as shown in Fig. 2.

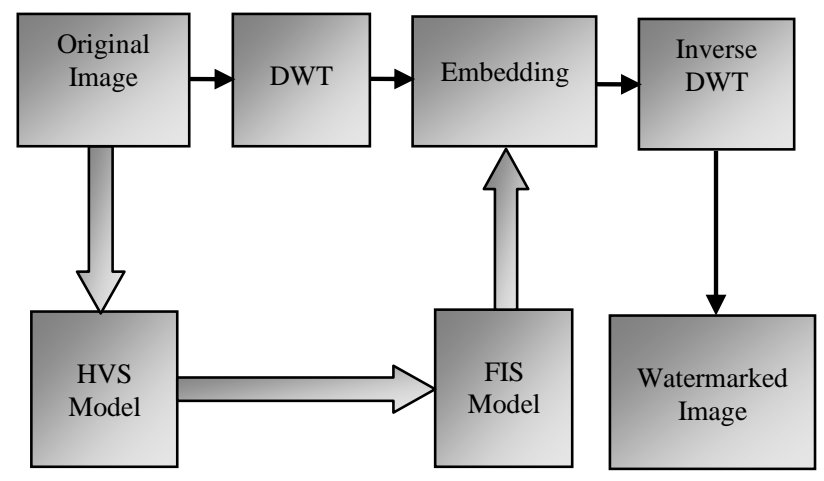

Fig.2. Block Diagram for Watermark Embedding

The steps for embedding are as follows:

1. Wavelet decomposition of the image to be watermarked up to three levels using a discrete wavelet transform as shown in Fig.3. In the first phase, image to be watermarked is decomposed by discrete wavelet 
transform. The first level discrete wavelet transform decomposes image in lower resolution approximation image (LL1) as well as horizontal (LH1), vertical (HL1), and diagonal (HH1) detail components. The sub-band LL1 containing major energy of image is the lowestfrequency component in the first level of decomposition.

2. Further, second level wavelet transform is applied to decompose $\mathrm{LL}_{1}$ band into sub-bands LL2, LH2, HL2, and $\mathrm{HH} 2$ and then 3-level for LL2 into sub-bands LL3, LH3, HL3, and HH3. DWT coefficients are obtained for each sub-band.

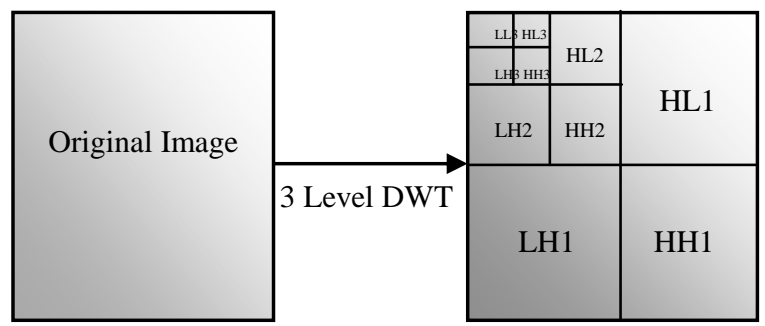

Fig.3. DWT Decomposition of Original Image using Three-Level Pyramid

3. The weight for sub-bands based on fuzzy rules, membership function and HVS parameters is determined. The larger the magnitude of wavelet coefficient, more significant it is. Magnitude of DWT coefficients is larger in approximation bands at each level of decomposition and is smaller for other detail bands.

4. Watermark is inserted in the form of binary logo, sign, text or a combination thereof by modifying significant wavelet coefficients at each level of decomposition structure.

5. HVS parameters such as brightness, texture and contrast are selected and computed for each sub-band by using approximate sub-band of the respective level.

6. Fuzzy inference system is built using three HVS parameters as fuzzy input variables wherein different fuzzy rules are derived from these parameters followed by assigning a suitable membership function to generate fuzzy output variable.

7. The Membership function decides mapping between input and output variables. Gaussian membership function is used in the proposed approach. The fuzzified output based on HVS characteristics is used for computing weight factor for each wavelet coefficient of the host image and insertion of watermark based on its weight. The inferred value is found by using centroid method of defuzzification.

8. The weight calculated is a local parameter which indicates maximum amount of modification that can be applied to a wavelet coefficient without any perceptual degradation.

9. The watermark embedding process first decomposes the original image into three levels using discrete wavelet transform. The local characteristics of the image are subsequently extracted using the model of the HVS, and the quantization step of DWT coefficients is then computed using the FIS in order to generate the watermark weighting function. Then, the logo watermark is inserted into the sub-bands.

The watermark insertion is performed as per equation (1).
$C^{\prime}$ band $(i, j)=C$ band $(i, j)+\alpha \beta(i, j) f(l, \theta) w(i, j)$

Where,

$\mathrm{C}$ band $(\mathrm{i}, \mathrm{j})$ : Original wavelet coefficient

$C^{\prime}$ band $(i, j):$ Watermarked wavelet coefficient

$\alpha$ : Watermark strength

$w(i, j)$ : Watermark signal

$\beta(i, j)$ : Weight of watermark

$f(l, \theta)$ : Sensitivity factor for frequency and orientation

The human eye sensitivity factor to frequency and orientation $\mathrm{f}(1, \theta)$ can be computed as given in equation (2). This equation takes into consideration that human visual system is less sensitive to disturbances in higher resolution bands and diagonal bands with $45^{\circ}$ orientation.

$$
f(l, \theta)=(\text { level }) \times(\text { theta })
$$

Where,

$$
\begin{aligned}
\text { theta } & =\sqrt{ } 2 \text { if } \theta=1 \\
& =1 \quad \text { otherwise } \\
\& \text { level } & =1.00 \quad \text { if } l=1 \\
& =0.32 \quad \text { if } 1=2 \\
& =0.16 \quad \text { if } l=3
\end{aligned}
$$

Weight of watermark $\beta(i, j)$ is computed from fuzzy inference system as shown in figure 4 .

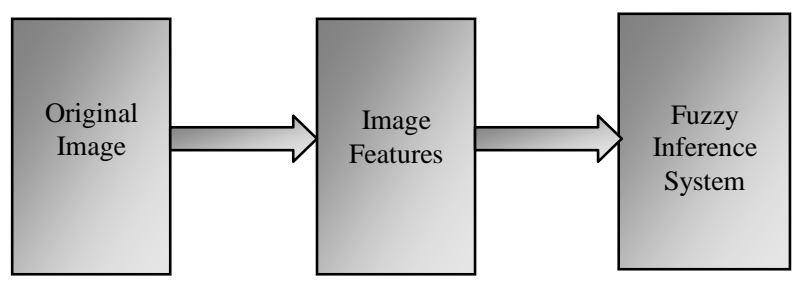

Fig.4. Computation of Weight of Watermark

10. Inverse discrete wavelet transform is applied to get watermarked image.

11. Objective analysis for imperceptibility is made using Peak Signal to Noise Ratio (PSNR). PSNR in decibel is calculated as follows [34]:

$$
\operatorname{PSNR}(d B)=10 \log _{10} \frac{255 \times 255}{M S E}
$$

Where, MSE is the Mean Square Error between original image and watermarked image. It is calculated as follows:

$$
M S E=\frac{1}{N t} \sum_{i, j}\left(X(i, j)-X^{\prime}(i, j)\right)^{2}
$$

Where,

$N t$ : Size of image,

$X(i, j)$ : Pixel value of original image \&

$X^{\prime}(i, j)$ : Pixel value of watermarked image. 
Human eyes cannot perceive the difference if PSNR is greater than $30 \mathrm{~dB}$. PSNR is measured to quantitatively evaluate the watermarked image quality.

\section{WATERMARK EXTRACTION PROCESS}

Watermark extraction and original image restoration is performed in this process at the receiving side. To realize the detection process, DWT is realized on watermarked image. Watermark detection is based on cross correlation between the original watermark and the extracted watermark $[35,36]$. The block diagram for watermark extraction is shown in figure 5 . The steps for extracting are as follows:

1. Subtraction of the watermarked and original image.

2. Calculation of normalized correlation coefficient (NC) and comparison with the threshold. $\mathrm{NC}$ is calculated to measure quality of extracted watermark. It is calculated as follows:

$$
\mathrm{NC}=\frac{\sum_{\mathrm{i}=0}^{\mathrm{p}-1} \sum_{\mathrm{j}=0}^{\mathrm{q}-1}\left(\mathrm{~W}_{\mathrm{ij}} \mathrm{W}^{\prime}{ }_{\mathrm{ij}}\right)}{\sum_{\mathrm{i}=0}^{\mathrm{p}-1} \sum_{\mathrm{j}=0}^{\mathrm{q}-1}\left(\mathrm{~W}_{\mathrm{ij}}\right)\left(\mathrm{W}_{\mathrm{ij}}\right)}
$$

Where,

$$
p \times q: \text { Size of watermark }
$$

$\left(\mathrm{W}_{\mathrm{ij}}\right)$ : Pixel value of watermark \&

$\left(\mathrm{W}^{\prime}{ }_{\mathrm{ij}}\right)$ : Pixel value of extracted watermark.

3. Decision on the presence or absence of watermark based on the threshold. If $\mathrm{NC}$ value is greater than threshold, it indicates existence of watermark. If $\mathrm{NC}$ is close to 1 , then extracted watermark is close to the original watermark.

4. If the watermarked image has not been subjected to malicious manipulation, the extracted watermark is visually recognizable pattern. The viewer can compare retrieved watermark with original watermark visually. This is a subjective measure.

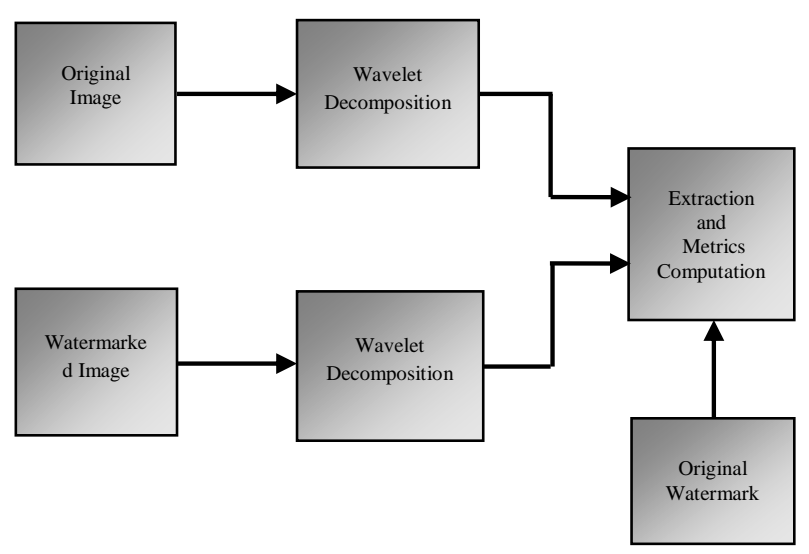

Fig.5 Block Diagram for Watermark Extraction

\section{EXPERIMENTAL RESULTS}

The algorithm is described for hiding a (32 x 32) binary logo image into a (256 x 256), 8 bits/pixel gray-level image. The watermark used for embedding is small compared to the size of host image. Watermark needs to be small as compared to original or host image in order to make it imperceptible. The performance of the proposed algorithm is tested on various images. It is noticed that this method is more suitable for photographic like gray scale images since they have more details in which watermark can be hidden. Watermark is embedded in luminance components rather than chroma components as later can be easily removed.

Bi-orthogonal wavelet filter is used for discrete wavelet transform. These wavelets represent family of compactly supported symmetric wavelets. The symmetry of filter coefficients is often desirable as it results in linear phase of the transfer function. The properties of perfect reconstruction and symmetry make bi-orthogonal wavelets suitable for watermarking. In these wavelets, two scaling and wavelet functions are used. One is used for analysis and the other is used for synthesis. Host image is decomposed into ten subbands using three levels of decomposition. Watermark image is embedded in sub-bands based on fuzzy inference system. FIS accepts three HVS inputs and applies different fuzzy rules to determine weight of watermark to be dynamically inserted in different regions of the host image. Out of all sub-bands, LL sub-band is having largest wavelet coefficients. The original and watermarked Lena images are shown in Fig. 6(a) and Fig. 6(b) respectively.

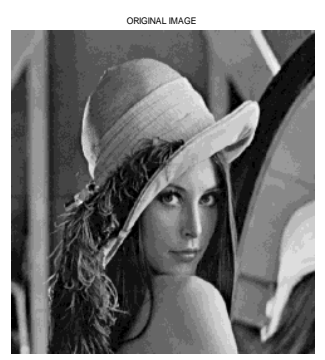

Fig. 6 (a) Original Lena

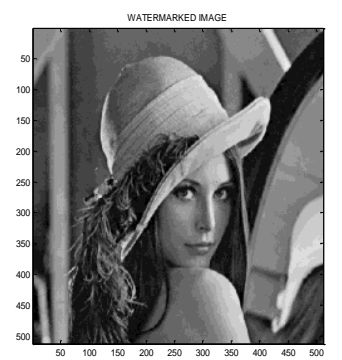

(b) Watermarked Lena
It can be observed that there is no perceptual degradation in watermarked image. PSNR value is calculated for objective analysis of watermarked image quality.

The minimum value of PSNR required for imperceptibility is 30dB. The watermarked Lena image shows PSNR value of $47.81 \mathrm{~dB}$. It can be observed that the algorithm can accurately model the regions in which the watermark insertion is performed, as it takes full advantage of the image adaptive HVS-FIS strategy.

Fig. 7(a) shows logo image embedded in the host image. This is 8-bit binary image of size $32 \times 32$. Fig. 7(b) shows the extracted logo image from the watermarked image using the proposed scheme. Extracted watermark can be easily recognized for ownership of image as against pseudo random binary watermark. Normalized coefficient is calculated for objective analysis of extracted watermark which is close to 1 .

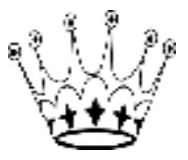

Fig. 7 (a) Original Logo Watermark

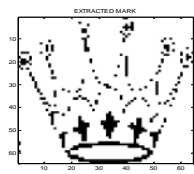

(b) Extracted Logo
To evaluate performance of the proposed watermarking scheme, different types of images are chosen for the test. These include cameraman, Elaine, Girl, Baboon and Peppers. The original images and watermarked images are shown in Fig. 8, Fig. 9, Fig. 10, Fig. 11 and Fig. 12. There are no visible artifacts between original images and watermarked 
images even though the characteristics of the host images vary from smooth images to textured images.

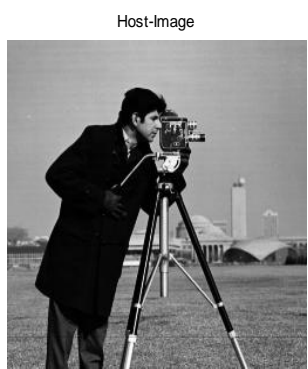

Fig. 8 (a) Original

\section{Cameraman}

Host-Image

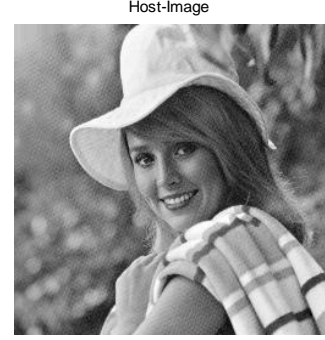

Fig. 9 (a) Original Elaine

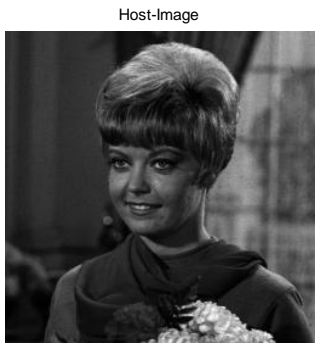

Fig. 10 (a) Original Girl

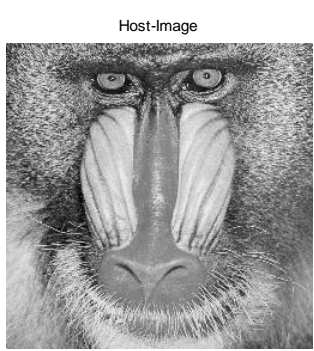

Fig. 11 (a) Original Baboon

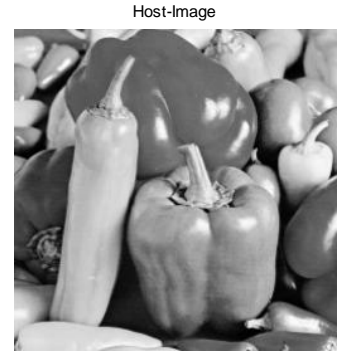

Fig.12. (a) Original Pepper
WATERMARKED IMAGE

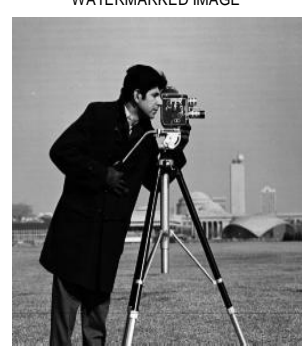

(b) Watermarked

\section{Cameraman}

WATERMARKED IMAGE

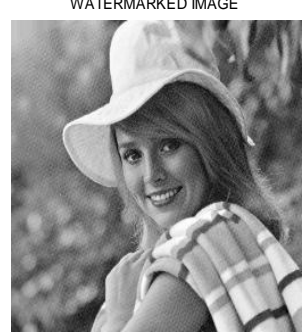

(b) Watermarked Elaine

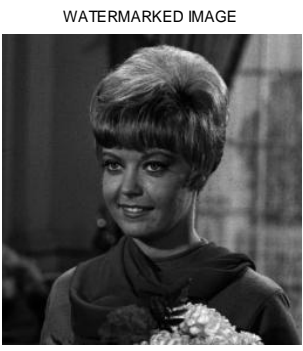

(b) Watermarked Girl

WATERMARKED IMAGE

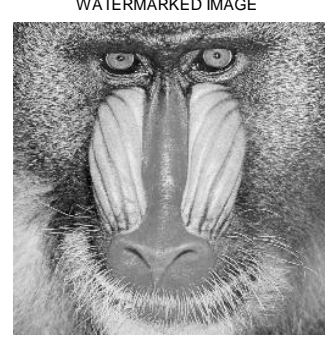

(b) Watermarked Baboon

WATERMARKED IMAGE

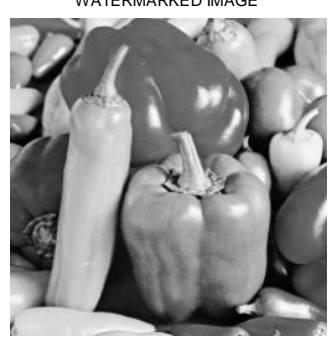

(b) Watermarked Pepper
Table 1shows PSNR values for all images are above 40dB. This indicates that watermarked image generated by the proposed method is imperceptible to human eyes.

Table 1. PSNR Values for Different Images

Proposed Method

\begin{tabular}{|c|c|c|}
\hline Sr. No. & Image & PSNR (dB) \\
\hline 1 & Lena & 47.81 \\
\hline 2 & Cameraman & 46.91 \\
\hline 3 & Elaine & 44.57 \\
\hline 4 & Girl & 49.43 \\
\hline 5 & Baboon & 49.32 \\
\hline 6 & Pepper & 43.63 \\
\hline
\end{tabular}

Table 2 summarizes the PSNR values for method using discrete cosine transform (DCT) for comparison with the PSNR values of the proposed method.

Table 2. PSNR Values for Different Images DCT Method

\begin{tabular}{|c|c|c|}
\hline Sr. No. & Image & PSNR (dB) \\
\hline 1 & Lena & 40.12 \\
\hline 2 & Cameraman & 40.23 \\
\hline 3 & Elaine & 39.87 \\
\hline 4 & Girl & 40.28 \\
\hline 5 & Baboon & 42.85 \\
\hline 6 & Pepper & 41.72 \\
\hline
\end{tabular}

Performance measurement of PSNR values for proposed scheme with the scheme using DCT is shown graphically in figure 13

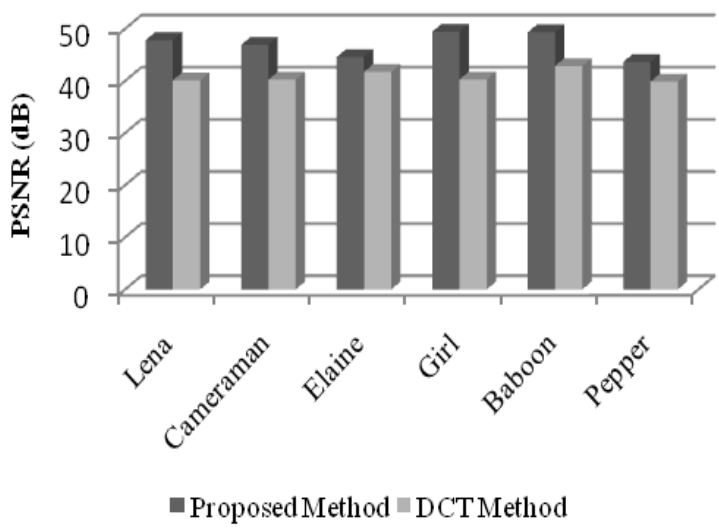

Fig. 13 Comparison of Performance of the two schemes

PSNR values for the proposed scheme are better, which shows better imperceptibility of the proposed scheme.

\section{CONCLUSION}

The proposed novel scheme is based on Human Visual System, Discrete Wavelet Transform and Fuzzy Inference system. A bi-orthogonal wavelet based logo watermarking technique for digital images is presented. Fuzzy inference system is the key component in this scheme. This scheme is 
capable of copyright protection of digital images by robust and imperceptible watermarking.

Watermark is embedded into the coefficients selected based on weight factors calculated by exploiting HVS characteristics. Experimental results demonstrate that this watermarking algorithm has good performance for imperceptibility of watermark. The performance of this approach can be improved by using different wavelet filters.

The proposed scheme finds its effective application in diverse fields. It can be applied for biometric data such as fingerprint,

iris or any other human identity. This scheme can be extended to colour images and 3-D images. It can be effectively used in video which is essentially a superset of images. The scheme finds its use in distance learning, digital libraries, digital TVs, e-commerce and e-governance.

\section{REFERENCES}

[1] Barni M, Bartolini F, Piva, "An Improved WaveletBased Watermarking Through Pixelwise Masking", IEEE transactions on image processing, Vol. 10, 2001 pp.783-791.

[2] Victor V., Guzman, Meana, "Analysis of a Waveletbased Watermarking Algorithm", IEEE Proceedings of the International Conference on Electronics, Communications and Computer, 2004, pp. 283-287.

[3] Zhao Dawei, Chen Guanrong, Liu Wenbo, "A Chaosbased Robust Wavelet-domain Watermarking Algorithm", IEEE International Conference on Multimedia and Expo (ICME), 2004.

[4] N. Kaewkamnerd and K.R. Rao, "Wavelet Based Image Adaptive Watermarking Scheme", IEEE Electronic Letters, Vol. 36,Feb. 2000, pp.312-313.

[5] Vaishali S. Jabade and Sachin R. Gengaje "Literature Review of Wavelet Based Digital Image Watermarking Techniques", International Journal of Computer Applications (0975 - 8887)Volume 31- No.1, October 2011,pp. 28-35.

[6] D. Kundur and D. Hatzinakos, "Digital Wateramrking using Multiresolution Wavelet Decomposition", Proceedings, IEEE International Conference Acoustic, Speech, Signal Processing,Vol. 5,1998, pp. 2969-2972.

[7] Miang Zuang Zang, Na Zang and Jian Guo Jiang, “An Adaptive Digital Watermarking Algorithm Based on Balanced Multi-wavelet", Proceedings, Fifth IEEE International Conference on Information Assurance ans Security,2009, pp. 243-246.

[8] Hey Jiang and Liu Jianjun, "A Digital Watermarking Algorithm for DEM Image Based on Stationary Wavelet Transform", Fifth IEEE International Conference on Information Assurance and Security, 2009, pp. 221-224.

[9] Peng Liu, Zhizhong Ding, “A Blind Image Watermarking Scheme Based on Wavelet tree Quantization", Second IEEE International Symposium on Electronic Commerce and Security, 2009, pp. 218222.

[10] Daxing Zhang, Zhigeng Pan and Haihua Li, "A Contour-based Semi-fragile Image Watermarking Algorithm in DWT Domain", Second IEEE
International Workshop on Education Technology and Computer Science, 2010, pp. 228-231.

[11] Wei Cao, Yixin Yan, Shengming Li, "Robust Image Watermarking Based on Singular Value Decomposition in DT-CWT Domain", IEEE International Workshop on Imaging Systems and Techniques, IST, 2009.

[12] Lin Zhuang "Multipurpose Digital Watermarking Algorithm Based on Morphological Wavelet Transform", IEEE International Conference on Communications and Mobile Computing, 2009, pp. 396-400.

[13] Peter Meerwald, Christian Koidl, and Andreas Uhl, "Attack on Watermarking Method Based on Significant Difference of Wavelet Coefficient Quantization", IEEE Transactions on Multimedia, Vol. 11, No. 5, August 2009, pp. 1037-1041.

[14] Der-Chyuan Lou , Hao-Kuan Tso, Jiang-Lung Liu "A Copyright Protection Scheme For Digital Images Using Visual Cryptography Technique", Computer Standards and Interfaces 29, 2007, pp.125-131.

[15] Santa Agreste, Guido Andaloro, Daniela Prestipino, Luigia Puccio, "An Image Adaptive Wavelet Based Watermarking of Digital Images", Journal of Computational and Applied Mathematics, 2006, pp. 19.

[16] Wen-Nung Lie, Guo-Shiang Lin, Sheng-Lung Cheng, "Dual Protection of JPEG Image Based on Informed Embedding And Two Stage Watermark Extraction Techniques", IEEE Transactions on information forensics and security, Vol. I, 2006, pp. 330-341.

[17] Enpingli, Huaqing Liang, Xinxin Niu, "Image Watermarking Scheme Based On Wavelet Tree Quantization Robust To Geometric Attacks", IEEE Proceedings of Sixth World Congress on Intelligent Control and Automation, pp. 10256-10260.

[18] Said E. El-Khamy, Fellow IEEE, Mona I. Lotfy and Rowayda A. Sadek, "A Block Based Wavelet Watermarking Technique for Copyright Protection and Authentication", IEEE Transactions, Vol.1, 2003, pp. 90-93.

[19] Serbia and Montenegro, "Copyright Protection using Wavelet Packets", IEEE Transactions, TELSIKS, 2005,

[20] Zhiquan Lu and Xiao-Ping Zhang,"Robust Image Watermarking Based on the Wavelet Contour Detection", IEEE Transactions, Vol.2, 2005, pp.11651168.

[21] Yusnita Yusof, Othman Khalifa, "Imperceptibility and Robustness Analysis of DWT- Based Digital Image Wateramrking", Proceedings of the International Conference on Computer and Communication Engineering, 2008, pp. 1325-1330.

[22] M.F. Fahmy and G. Fahmy, “A Quasi Blind Watermark Extraction of watermarked Natural Preserve Transform Images", IEEE Ineternational Conference on Image Processing,2009, pp.3665-3668.

[23] Dr. M.A. Dorairangaswamy, B. Padmavathi, "An Effective Blind Watermarking Scheme for Protecting Rightful Ownership of Digital Images", IEEE, TENCON, 2009, pp. 1-6. 
[24] Ming-Xiang Zang, Na Zang, Jian-guo Jiang, “An Adaptive Digital Watermarking Algorithm Based on Balanced Multi-wavelet", Fifth international Conference on Information Assuirance and Security, 2009, pp. 243-246.

[25] Gui Xie (2004),"Robust Wavelet-Based Blind Image Watermarking Against Geometrical Attacks", IEEE International Conference on Multimedia and Expo (ICME),Vol.3, 2004, pp. 2051-2054.

[26] Santi P. Maity and Seba Maity, "Multistage Spread Spectrum Watermark Detection Technique using Fuzzy Logic", IEEE Signal Processing Letters, Vol.16, No.4, 2009, pp. 245-248.

[27] Ming-shing Hsieh, "Image Watermarking based on Fuzzy Inference Filter", IEEE Proceedings of the International Conference on Machine Learning and Cybernetics, Baoding, 2009, pp.3058-3063.

[28] Prof. Sharvari Tamane, Dr. R.R. Manza, Dr. R.R. Deshmukh, "3D ModelsWatermarking using Fuzzy Logic", International Conference on Advances in Computing Technologies, 2009, pp. 195-197.

[29] D.C. Lou and T.L. Yin, "Adaptive Digital Watermarking using Fuzzy Clustering Technique", IEICE Transactions on Fundamentals, Vol. E84-A, Aug.2001, No.8,pp.2052-2060.

[30] Chip Hong Chang and Mingyan Zhang, "Fuzzy-Art based adaptive digital watermarking scheme", IEEE Transactions on Circuits and Systems for Video Technology, Vol. 15, no. 1, 2005, pp. 65-81.
[31] Nizar Sakr, Jiying Zhao, and Voicu Groza , "A Dynamic Fuzzy Logic Approach to Adaptive HVSbased Watermarking", IEEE international workshop on Haptic Audio Visual Environments and their Applications, 2005, pp. 121-126.

[32] Ming-shinghsieh and Din-changtseng, "Image SubBand Coding Using Fuzzy Inference and Adaptive Quantization", IEEE Transactions on Systems, Man and Cybernetics, Vol.33, 2003, pp. 509-513.

[33] Chip Hong Chang and Mingyan Zhang, "Fuzzy-Art based adaptive digital watermarking scheme", IEEE Transactions on Circuits and Systems for Video Technology, Vol. 15 no. 1, 2005, pp. 65-81.

[34] Yuanhai Shao, Wei Chen, Chan Liu, "Multiwaveletbased Digital Watermarking with Support Vector Machinr Technique", IEEE Conferenc, CCDC,2008, pp.4557-4561.

[35] A. Adhipati Reddy, B. N. Chatterji, "A New Wavelet Based Logo Watermarking Scheme", Science Direct, Pattern Recognition Letters, 26, 2004, pp.1019-1027.

[36] B.N. Chatterji, Manesh Kokare, A. Adhipathi Reddy, Rajib Kumar Jha., "Wavelets for Content Based Image Retrieval and Digital Watermarking for Multimedia Applications", IEEE Transactions, Vol.2, 2003, pp.812816. 